\title{
Erratum to: Arachidonic acid induces acetyl-CoA carboxylase 1 expression via activation of CREB1
}

\author{
Yin Peng $\cdot$ Ting Lei $\cdot$ Jihong Yuan $\cdot$ Xiaodong Chen • \\ Qinqiang Long $\cdot$ Jian Zhang $\cdot$ Peng Lei $\cdot$ Bin Feng • \\ Zaiqing Yang
}

Published online: 5 November 2009

(C) Humana Press 2009

Erratum to: Endocr

DOI 10.1007/s12020-009-9241-8

In the original publication, the name of the sixth author in the list is misspelled. The correct spelling is Jian Zhang.

The online version of the original article can be found under doi: 10.1007/s12020-009-9241-8.

Y. Peng · T. Lei · J. Yuan · X. Chen · Q. Long · J. Zhang ·

P. Lei $\cdot$ B. Feng $\cdot$ Z. Yang $(\bowtie)$

Key Laboratory of Agricultural Animal Genetics, Breeding and Reproduction of Ministry of Education, College of Life Science and Technology, Huazhong Agricultural University, 430070

Wuhan, People's Republic of China

e-mail: yangzq@mail.hzau.edu.cn

Y. Peng

e-mail: py_rimidine@yahoo.com.cn 